\title{
Does music performance allude to locomotion? A model of final ritardandi derived from measurements of stopping runners $^{\text {a) }}$
}

\author{
Anders Friberg ${ }^{\text {b) }}$ and Johan Sundberg ${ }^{\text {b) }}$ \\ Royal Institute of Technology, Speech, Music, and Hearing, SE-100 44 Stockholm, Sweden
}

(Received 20 November 1997; revised 30 September 1998; accepted 22 October 1998)

\begin{abstract}
This investigation explores the common assumption that music and motion are closely related by comparing the stopping of running and the termination of a piece of music. Video recordings were made of professional dancers' stopping from running under different deceleration conditions, and instant values of body velocity, step frequency, and step length were estimated. In decelerations that were highly rated for aesthetic quality by a panel of choreographers, the mean body velocity could be approximated by a square-root function of time, which is equivalent to a cubic-root function of position. This implies a linear relationship between kinetic energy and time, i.e., a constant braking power. The mean body velocity showed a striking similarity with the mean tempo pattern of final ritardandi in music performances. The constant braking power was used as the basis for a model describing both the changes of tempo in final ritardandi and the changes of velocity in runners' decelerations. The translation of physical motion to musical tempo was realized by assuming that velocity and musical tempo are equivalent. Two parameters were added to the model to account for the variation observed in individual ritardandi and in individual decelerations: (1) the parameter $q$ controlling the curvature, $q=3$ corresponding to the runners' deceleration, and (2) the parameter $v_{\text {end }}$ for the final velocity and tempo value, respectively. A listening experiment was carried out presenting music examples with final ritardandi according to the model with different $q$ values or to an alternative function. Highest ratings were obtained for the model with $q=2$ and $q=3$. Out of three functions, the model produced the best fit to individual measured ritardandi as well as to individual decelerations. A function previously used for modeling phrase-related tempo variations (interonset duration as a quadratic function of score position) produced the lowest ratings and the poorest fits to individual ritardandi. The results thus seem to substantiate the commonly assumed analogies between motion and music. (C) 1999 Acoustical Society of America.
\end{abstract}

[S0001-4966(99)01402-2]

PACS numbers: 43.10.Ln, 43.75.St [WJS]

\section{INTRODUCTION}

Music performance is often regarded as an area of almost unlimited variability; different artists play the same piece of music in very different ways. On the other hand, our attempts to synthesize music performance (e.g., Friberg, 1995a; Sundberg, 1988) often demonstrated that if a given performance parameter was varied beyond a narrow range, musically unacceptable performances were obtained. Most musicians agree on the importance of, e.g., finding the right tempo for a piece. Also, in many cases, a consensus about various performance parameters can be reached within an ensemble. What is the basis for such agreement? Why is the exact value or change of tempo so important to what is musically acceptable? Is it possible that we use a common reference taken from our extramusical experiences? Two candidates for such a reference have been suggested: speech and motion. By comparing the stopping of running with the final ritardando, this article tests the hypothesis that tempo changes allude to locomotional patterns.

Music is commonly associated with motion. This is

\footnotetext{
a) "Selected research articles" are ones chosen occasionally by the Editorin-Chief that are judged (a) to have a subject of wide acoustical interest, and (b) to be written for understanding by broad acoustical readership.

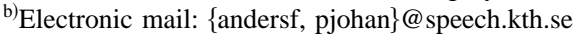

manifested by the common use of motion words in descriptions of music, such as lento, andante, corrente (slow, walking, running). Truslit (1938) even suggested that the performer must imagine an inner motion in order to produce a good performance and that the listener must "hear" this inner motion in order to appreciate the music: "Every crescendo and decrescendo, every accelerando and decelerando, is nothing but the manifestation of changing motion energies, regardless of whether they are intended as pure movement or as expression of emotion." [Cited from the English synopsis by Repp (1993).] The connection between music and motion has been discussed in a relatively intuitive way in numerous articles and essays partially reviewed by Shove and Repp (1995).

An experimental attempt to test the possibility of a direct connection between music and motion was done by Sundberg et al. (1992). The vertical-force patterns exerted by the foot on the ground were measured for different gaits and were then translated to sound-level envelopes of tones. These tones were repeated in sequences with different tempos and evaluated in three listening experiments. In one experiment, the listeners were asked to describe the examples in any terms. Responses relating to motion occurred in up to $50 \%$ of the total number of responses in some cases, and was more common (1) when the tone interonset time was the same as 
the original step interonset time and (2) at tempos corresponding to about $600-\mathrm{ms}$ interonset time. In another experiment, the listeners rated the sequences along visual analogue scales for each of 24 motion adjectives. The result showed that the tone sequences were classified in qualitative accordance with the original gaits. Thus, information on locomotion characteristics could be transferred to listeners by using the force patterns of the foot as sound-level envelopes.

This investigation represents another attempt to directly link locomotion to music performance. The overall strategy was to find out if the tempo changes in final ritardandi can be described by the same mathematical model as the velocity changes during runners' stopping. The article first reviews mathematical representations of tempo and different models that have been used for describing tempo variations in music performance. Then, measurements and visual assessments of runners' decelerations are presented and compared with previous measurements of final ritardandi. After this, a mathematical model of the final ritardando, derived from the runners' data, is presented and evaluated. Finally, this model is applied to individual cases of runners' decelerations and of final ritardandi.

\section{BACKGROUND}

\section{A. Representation of tempo}

Different strategies for representing tempo data have been used in the past. Most researchers have used score position as the independent variable. In other words, each note value is represented by a corresponding distance along an axis representing score position. For example, a whole note is represented by the distance 1 , a half note by the distance $1 / 2$, etc. Alternatively, the shortest note value occurring in the piece is used as the unit for the score position axis. Thus, if the shortest unit is a 16th note, the distance of 16 will represent a whole note. The commonly used terms nominal duration and deadpan duration are both used for durations that exactly correspond to note values and are thus equivalent to score position.

Another possibility is to use time for the independent variable. This was recommended by Todd $(1992,1995)$, who argued that ongoing time is more easily perceived than the more abstract score position. There is, however, a computational problem when a given tempo curve, expressed as a function of time, is applied to a music example that by definition is expressed in terms of score position. Therefore, it is necessary to transform the tempo curve into a function of score position. Unfortunately, such transformations have an analytic solution only for some simple mathematical expressions.

A dependent variable tempo, defined as the inverse of tone interonset interval (IOI), seems a natural choice. However, beat or tone IOI have also been used.

Figure 1 shows some simple ritardando curves and how they appear in three different representations. The left column of panels in Fig. 1 shows tone IOI as a function of score position $x$, the middle column of panels shows the same curves transformed into tempo as a function of score position, and the right column of panels shows the same curves (a) Quadratic 101 of $x$
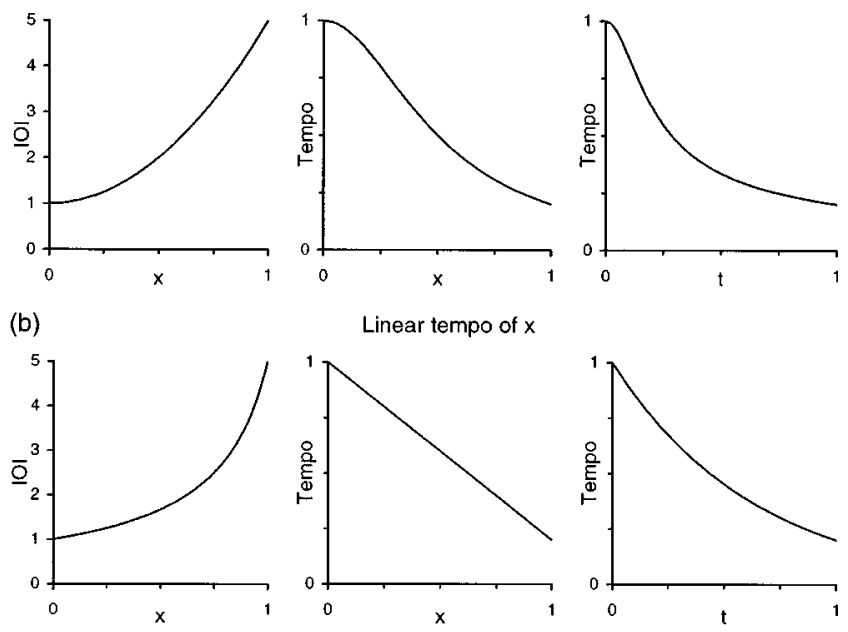

Linear tempo of $x$

(c)
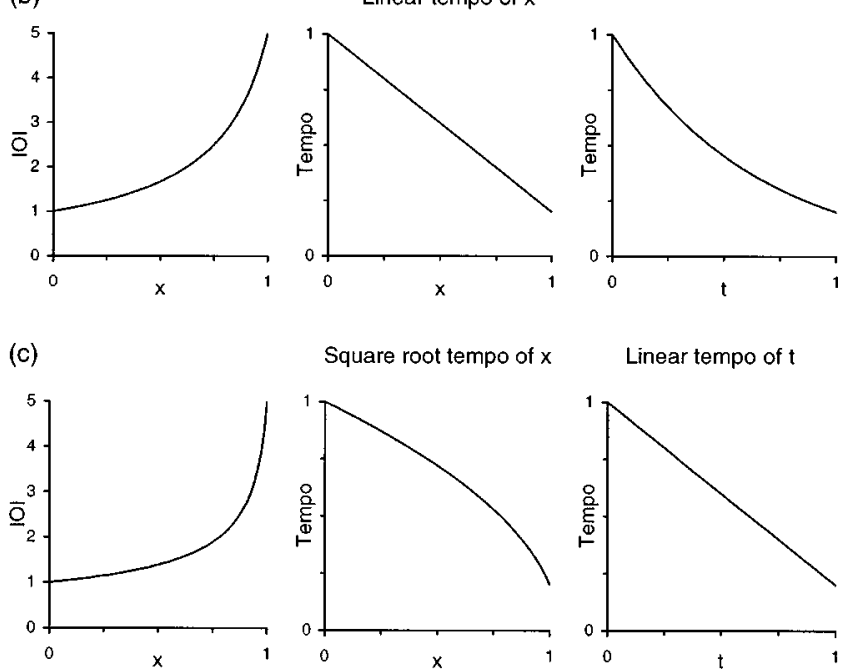

Square root tempo of $x$

Linear tempo of $t$
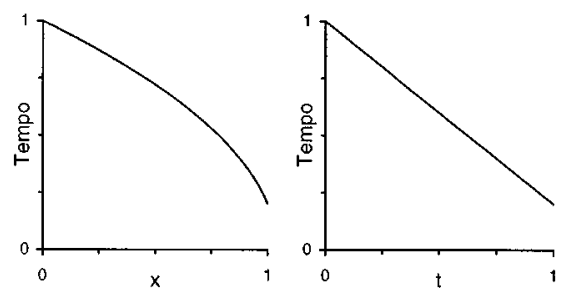

(d)

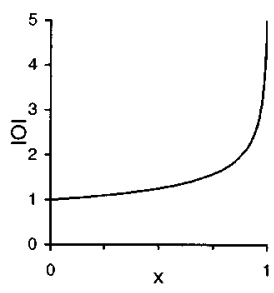

Cubic root tempo of $x$

Square root tempo of $t$
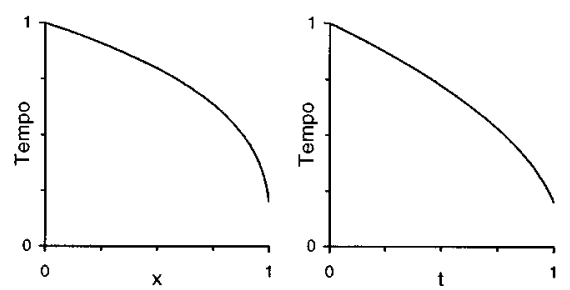

FIG. 1. Comparison of different methods of representing tempo changes during the final ritardando using the indicated four simple functions [(a)(d)]. The columns show these functions plotted against alternative abscissas: IOI as a function of position $x$ (left column), tempo as a function of position $x$ (middle column), and tempo as a function of time $t$ (right column).

expressed as tempo as a function of time. The choice of variables obviously affects the shape of the curve profoundly. A change of the independent variable from score position to time (mid- and right columns) results in a more concave or less convex curvature. For example, when the tempo is a square-root function of score position, it becomes a linear function of time. A quadratic relation between IOI and score position (top-left panel) has been used several times in the past (e.g., Todd, 1985; Repp 1992b; Friberg, 1995b). Note that this curve, when transformed into a curve showing tempo versus score position (top-middle panel), starts with a convex and ends with a concave curvature.

\section{B. Previous models of tempo variations}

Sundberg and Verrillo (1980) analyzed the characteristics of the final ritardando in music performances. They measured tone IOIs in 24 performed final ritardandi in recordings of baroque music and computed an average final ritardando profile for tempo versus time. Out of these data, they derived a model consisting of two phases, each of which 
showed a linear decrease of tempo when expressed as a function of score position. The length of the second phase corresponded to the last musical motif of the piece.

Kronman and Sundberg (1987) found that the average final ritardando profile could be rather accurately approximated by one single square-root function, thus abandoning the two-phase model. They further related this function to what they assumed to be an accurate description of the decrease of the footstep frequency of a stopping runner, provided there was constant body deceleration and constant step length. This similarity suggested an interesting analogy between musical tempo and step frequency. It may be noted that the step frequency is equivalent to velocity if the step length is constant. However, Kronman and Sundberg used the ritardando data by Sundberg and Verrillo, expressed as instant tempo versus time, and compared these data with the deceleration model expressed as step frequency versus position. A simpler analogy would be to use time as the independent variable in both cases. If this is done, the tempo curve for the final ritardando becomes much less similar to the model, as in this case the step frequency comes out as a linear function of time.

Feldman et al. (1992) investigated curves of performed accelerandi and ritardandi in five examples that were selected by David Epstein from commercially available recordings. The authors developed a simple force model of physical motion that assumed tempo to be equivalent with velocity. They regarded smooth beginnings and endings important characteristics of such tempo changes, and therefore proposed that tempo should be expressed as a quadratic or cubic function of time. These functions correspond to a linear and a quadratic change of force with time, respectively. However, in the subsequent analysis, they used beat duration as a function of score position instead of tempo as a function of time, thus suggesting that these two completely different representations could be regarded as equivalent. They fitted their data, expressed in this new form (beat duration of score position), to linear, quadratic, or cubic functions. They found the two latter alternatives reasonably appropriate to approximate these data and falsely concluded that their force model neatly accounted for the observed tempo profiles.

Repp (1992a) measured the timing of 28 performances of Robert Schumann's Träumerei. The accelerandoritardando shape of a salient, six-note motif was successfully modeled by expressing tone IOI as a quadratic function of score position. He also carried out a perceptual evaluation of this function applied to synthesized piano performances of the same music example (Repp, 1992b).

Todd (1985) presented a model of phrase-related tempo changes in music performances. In this model, tone IOIs were expressed as quadratic functions of score position. Later, he proposed a modified version (Todd, 1992, 1995) based on an analogy between velocity in physical motion and tempo in music performance, thus implying equivalence between physical position and score position. He simply assumed the deceleration and acceleration forces to be constant, which implied linear variations of tempo as a function of time (see also Longuet-Higgins and Lisle, 1989). It can be noted that this case corresponds to a square-root function of
TABLE I. Data on the dancers who performed the analyzed decelerations.

\begin{tabular}{clcc}
\hline \hline Dancer & Sex & Height $(\mathrm{cm})$ & Weight $(\mathrm{kg})$ \\
\hline M1 & Male & 181 & 75 \\
M2 & Male & 180 & 67 \\
F1 & Female & 170 & 49 \\
F2 & Female & 165 & 53 \\
\hline \hline
\end{tabular}

score position [Fig. 1(c) middle- and right panels], thus similar to the model presented by Kronman and Sundberg (1987).

Building on Todd's idea of a quadratic relation between tempo and score position, coauthor A.F. developed a model that related tempo changes to the hierarchical phrase structure (Friberg, 1995b). The model allowed for variations that typically can be observed between different experts' performances of the same piece. Thus, when tested against Repp's measurements of the 28 performances of Träumerei, the model successfully accounted for the tempo variation at the phrase level observed among these performances.

Summarizing, the models above have described the shape of the tempo variations as (1) a linear change of tempo versus score position, (2) as a quadratic change of IOI versus score position, or (3) as a linear change of tempo versus time. These models were based merely on assumptions regarding physical motion. Thus, no data were collected from real decelerations as performed by, e.g., human runners. This lack of reference to motion data was pointed out by Desain and Honing (1996), who also mentioned the likely influences of musical structure and global tempo on the final ritardando. Although human locomotion has been studied in several investigations (Margaria, 1976; Cavagna et al., 1988; Alexander, 1995; Nilsson and Thorstensson, 1987; Ae et al., 1992), the characteristics of stopping locomotion seem to have attracted little attention. Hence, it seemed necessary to perform actual measurements of runners' decelerations.

\section{RUNNERS' DECELERATION}

\section{A. Methods}

\section{Deceleration measurements}

Professional dancers, two males and two females, were used as subjects (Table I). Their task was to run at predetermined initial-step frequencies along a marked straight line and to stop their running at a marked point along this line. The subjects were not asked to think of any musical models for their running and deceleration; actually, music was not mentioned during the instruction of the subjects. Although the subjects were professional dancers, neither their running nor their decelerations appeared special in any sense.

The initial step frequencies were given in terms of acoustical click signals provided by a metronome connected to a loudspeaker. This signal was presented before the subjects started to run and continued during the first part of their running. Two step frequencies were selected, 2.9 and $4 \mathrm{~Hz}$ (interstep intervals 340 and $250 \mathrm{~ms}$, respectively). The point at which the subjects were supposed to start the slowing down was either free, i.e., left to the subjects to decide, or 
shown by a mark on the floor, 4 or $7 \mathrm{~m}$ from the end point. Each condition was recorded five times, but only two of the subjects used the free condition.

The experiment was carried out in a large hall. Stripes of sticky reflective tape were fastened to the floor perpendicular to the direction of running, 1-m apart at the beginning of the running path, and at $0.1 \mathrm{~m}$ along the final $10 \mathrm{~m}$. Such tape stripes were also fastened to the subjects' ankles and hips as references. The footsteps were picked up by an audio microphone and also by a set of accelerometers placed on the floor at 2-m distances along the final $10 \mathrm{~m}$ of the running path. The audio and accelerometer signals were recorded on a TEAC PCM data recorder. The running was filmed by a video camera and recorded on a VHS recorder.

By displaying the video recording frame by frame, the step length could be determined with an accuracy of approximately $\pm 3 \mathrm{~cm}$. The audio and accelerometer recordings were analyzed using a waveform editing program (Soundswell Signal Workstation) allowing an accuracy of about $\pm 5 \mathrm{~ms}$ in determining the time intervals between the impacts of the footsteps.

\section{Rating experiment}

Although the runners were all professional dancers, it seemed unrealistic to preclude that they invariably performed decelerations that completely corresponded to their artistic intentions. As it seemed unwise to base a model of final ritardandi on accidentally pathological examples of runners' decelerations, an evaluation experiment was run. Using the video recordings from the experimental session, an initial assessment of the aesthetical overall quality of the decelerations was carried out by a highly experienced teacher of eurhythmics at the Royal College of Music, Stockholm. The purpose of this pretest was to reduce the number of decelerations in the subsequent analyses.

Eight decelerations from each of the two step frequencies were selected. The selection was made so as to obtain a maximum variation with regard to subject, rating values obtained in the pretest, and deceleration-distance conditions. These 16 decelerations were copied into a video test tape, where each deceleration appeared three times. They were separated by 7 -s pauses. The test took about 15 min and was preceded by eight practice trials. The tape was presented to six experienced experts in choreography and/or eurhythmics. The judges were asked to rate overall aesthetic quality of the final deceleration, taking into consideration form and balance. For each of the 48 decelerations, the judges put a mark on a 10-cm line on an answer sheet where the left end represented "Extremely unaesthetic', and the right end "Extremely aesthetic."

\section{B. Results}

\section{Rating experiment}

Table II summarizes the test result in terms of ratings averaged across replications and judges and the corresponding standard deviations. The table shows that the judges did not clearly prefer any particular step frequency or deceleration distance.
TABLE II. Ratings, averaged across repetitions and judges, of the aesthetical quality of the decelerations, listed in rank order.

\begin{tabular}{cccccc}
\hline \hline Runner & $\begin{array}{c}\text { Initial-step } \\
\text { frequency } \\
\text { (Hz) }\end{array}$ & $\begin{array}{c}\text { Deceleration } \\
\text { distance condition } \\
(\mathrm{m})\end{array}$ & $\begin{array}{c}\text { Mean } \\
\text { rating } \\
(\mathrm{cm})\end{array}$ & $\begin{array}{c}\text { s.d. } \\
(\mathrm{cm})\end{array}$ & Rank \\
\hline M1 & 2.9 & 7 & 6.3 & 2.5 & 1 \\
M1 & 2.9 & 4 & 6.3 & 2.2 & 2 \\
M1 & 4 & 7 & 6.2 & 2.5 & 3 \\
F2 & 2.9 & free & 5.9 & 2.5 & 4 \\
F2 & 2.9 & free & 5.8 & 2.5 & 5 \\
F2 & 4 & free & 5.8 & 1.9 & 6 \\
F2 & 4 & free & 5.6 & 2.7 & 7 \\
F1 & 2.9 & free & 5.3 & 2.4 & 8 \\
F1 & 2.9 & free & 5 & 2.3 & 9 \\
F2 & 4 & 4 & 4.8 & 2.1 & 10 \\
F1 & 4 & free & 4.4 & 2.5 & 11 \\
F1 & 4 & free & 4.2 & 2.3 & 12 \\
M2 & 2.9 & 7 & 3.5 & 2.3 & 13 \\
M2 & 4 & 7 & 2.7 & 1.8 & 14 \\
M2 & 2.9 & 4 & 2.6 & 2 & 15 \\
M2 & 4 & 4 & 1.5 & 1.8 & 16 \\
\hline \hline
\end{tabular}

Figure 2 shows the ordered-mean ratings for all the decelerations. The difference in mean rating was small between the stimuli that were rated high and greater between those which rated low. Thus, the curve showed a slight trend to a discontinuity between rank-order numbers 12 and 13. One male dancer, M2, produced all four lowest-rated decelerations. Most of these four decelerations exhibited irregularities in step-frequency patterns during the deceleration, as seen in the right-top graph in Fig. 3, and were omitted in the computation of average decelerations.

\section{Deceleration patterns}

Figure 3 shows instant-step frequency (inverse of interstep duration) and the associated step length for the two decelerations that the judges rated highest. To give an idea of the variation in the data, the two lowest-rated decelerations are also included in this figure. The decelerations mostly included six to ten steps. The step-frequency patterns varied widely among the 16 decelerations, some decreasing slightly,

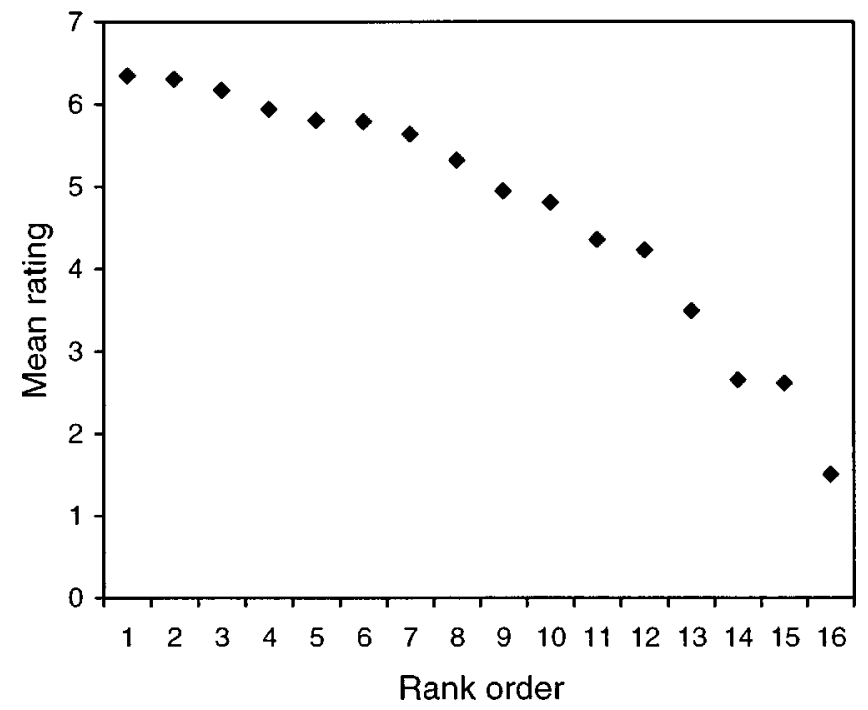

FIG. 2. Rank-ordered mean ratings obtained from the experiment where experts rated the aesthetical quality of runners' decelerations. 

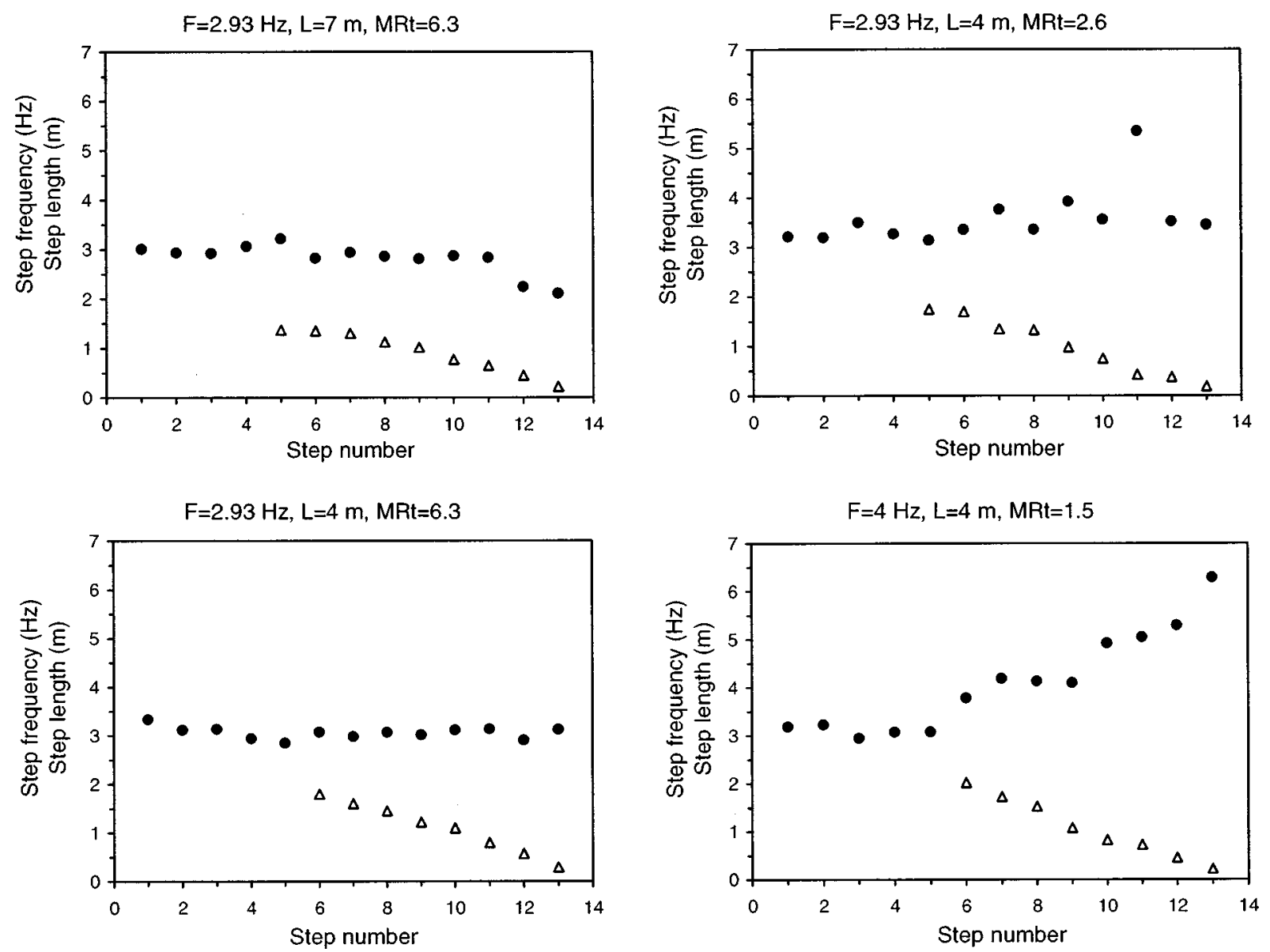

FIG. 3. Instant-step frequency (filled circles) and step-length (open triangles) patterns for the two highest- (left) and the two lowest- (right) rated decelerations. The initial values of step frequency $(F)$, deceleration length $(L)$, and the mean rating (MRt) of the deceleration are shown above each panel.

some remaining basically constant, and some even increasing toward the end of the deceleration, thus clearly negating the possibility that the change in step frequency serves as the model for the tempo change in final ritardandi. The step length, by contrast, decreased gradually toward the end of the deceleration in all cases. Thus, the slowing down was achieved mainly by decreasing the step length, an observation which is in good agreement with previous findings on the relation between step length and speed of locomotion (Alexander and Maloiy, 1984; Cavagna et al., 1988).

Figure 4 shows velocity versus distance to the point of stop for the same decelerations as in Fig. 3. Instant velocity was estimated at each step by dividing the step length with the step duration. The position of each velocity estimation was taken as the middle value between two step positions. The number of velocity estimations ranged from six to eight points and the estimated deceleration distance ranged from 5.9 to $8.2 \mathrm{~m}$ in the 12 decelerations. The normalized velocity versus normalized position for all 16 decelerations are shown in the Appendix, Fig. A2.

As the number of velocity data for each deceleration was comparatively small, an average was computed for the 12 highest-ranked decelerations (mean ratings $>4$ ). Two average-velocity curves were computed: one curve with position and one curve with time as the independent variable. In both cases, each of the 12 velocity curves was normalized with respect to the first estimated-velocity value and with respect to the associated position and time, respectively. Velocity values between data points were obtained by linear interpolation. The means were computed by sampling these interpolated normalized curves at 20 equidistant steps of position or time.

The resulting normalized mean velocity as a function of normalized position is shown in Fig. 5(a). The values of 0 and 1 on the abscissa correspond to the starting and the termination of the deceleration, respectively. The bars mark the 95\%-confidence interval for the estimation of the mean at the sampled positions.

The mean-velocity pattern, when plotted as a function of normalized time, is very similar to that of the normalized mean ritardando curve of Sundberg and Verrillo (1980), see Fig. 5(b). This supports previous suggestions that there is equivalence between velocity and tempo (Todd, 1992, 1995; Feldman et al., 1992). Note that, for reasons of comparison, the abscissa is normalized time. The match was obtained after scaling the abscissa of the velocity curve by a factor of 1.13. This value was found on a trial-and-error basis. The use of a scaling factor is motivated by the fact that, obviously, a ritardando curve can never reach a normalized tempo value of zero since it corresponds to an infinite note duration.

Kronman and Sundberg (1987) assumed that the deceleration force is constant during a deceleration of running (see also Todd, 1995). This implies that velocity is a straight line if time is used as the independent variable. As seen in Fig. 

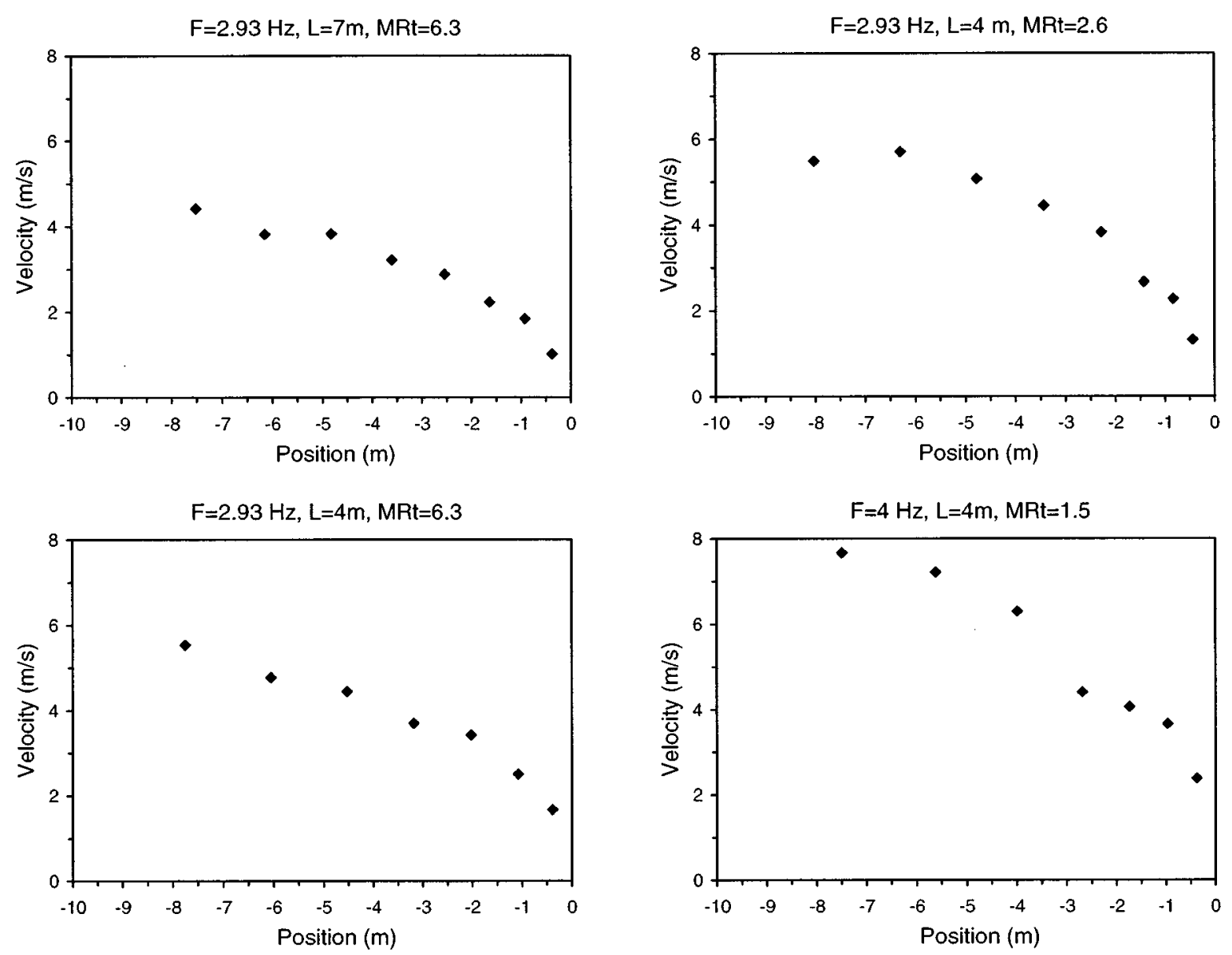

FIG. 4. Instant-velocity patterns for the decelerations shown in Fig. 3. The initial values of step frequency $(F)$, deceleration length $(L)$, and the mean rating (MRt) of the deceleration are shown above each panel.

5(b), this was not the case. However, the square of the velocity, i.e., the kinetic energy, is close to a straight-line function of time as shown in Fig. 5(c). Note that the straight-line approximation of the data point passes very close to the corners of the square in this figure. The close agreement between the data points and the line implies that the deceleration power was approximately constant throughout the entire deceleration. Individual velocity curves will be analyzed below in terms of how well our model fits the data.

\section{Discussion of decelerations}

An important question is to what extent the obtained velocity curves were specific to the experimental conditions in the recording session. Obviously we would have obtained quite different deceleration curves if the dancers had been running on a slippery surface, such as ice. The recordings were made in a gymnasium, where the floor has a comparatively large friction and thus should not have caused any abnormality in the deceleration data. Furthermore, in the rating experiment no preference was observed for any specific condition regarding step frequency or deceleration distance. Thus, our mean-velocity curve can be assumed to be reasonably representative for the stopping of running.

It could be argued that dancers are biased subjects in an experiment with the present aim, being prone to adopt the typical patterns of classical choreographic art. On the other hand, in the video recordings no tendency to use any special locomotion patterns could be seen. It could also be argued that professional dancers are a typical subjects as they have been specially trained to move in synchrony with music. However, professional dancers share with almost anyone the experience of moving to music, since dancing is a very commonly enjoyed form of social life.

To rate the aesthetical quality of a runner's deceleration implies the task of giving a global assessment of a stimulus possessing many dimensions, such as coordination of limb movements, posture, etc. (Todd, 1983). In our case, this would have contributed to the comparatively high standard deviations of the ratings. The judges may have attributed different degrees of importance to various aspects of a given deceleration. Still, the rating experiment seemed to serve its main purpose, viz., to identify decelerations that appeared particularly unaesthetical to expert judges.

When plotted in rank order, the distribution of the ratings showed a slight trend to an elbow-like curve. It is interesting that a similar curve was found when, in a quite different experiment, experts rated hoarseness and other voice characteristics in children's voices (Sederholm et al., 1993). In both these rating experiments, the notion of acceptability/ unacceptability of a multidimensional stimulus was probably relevant.

\section{MODEL}

A basis for the construction of our model for final ritardandi was the assumption that the deceleration power was constant in a runner's deceleration (vide supra). This implies 

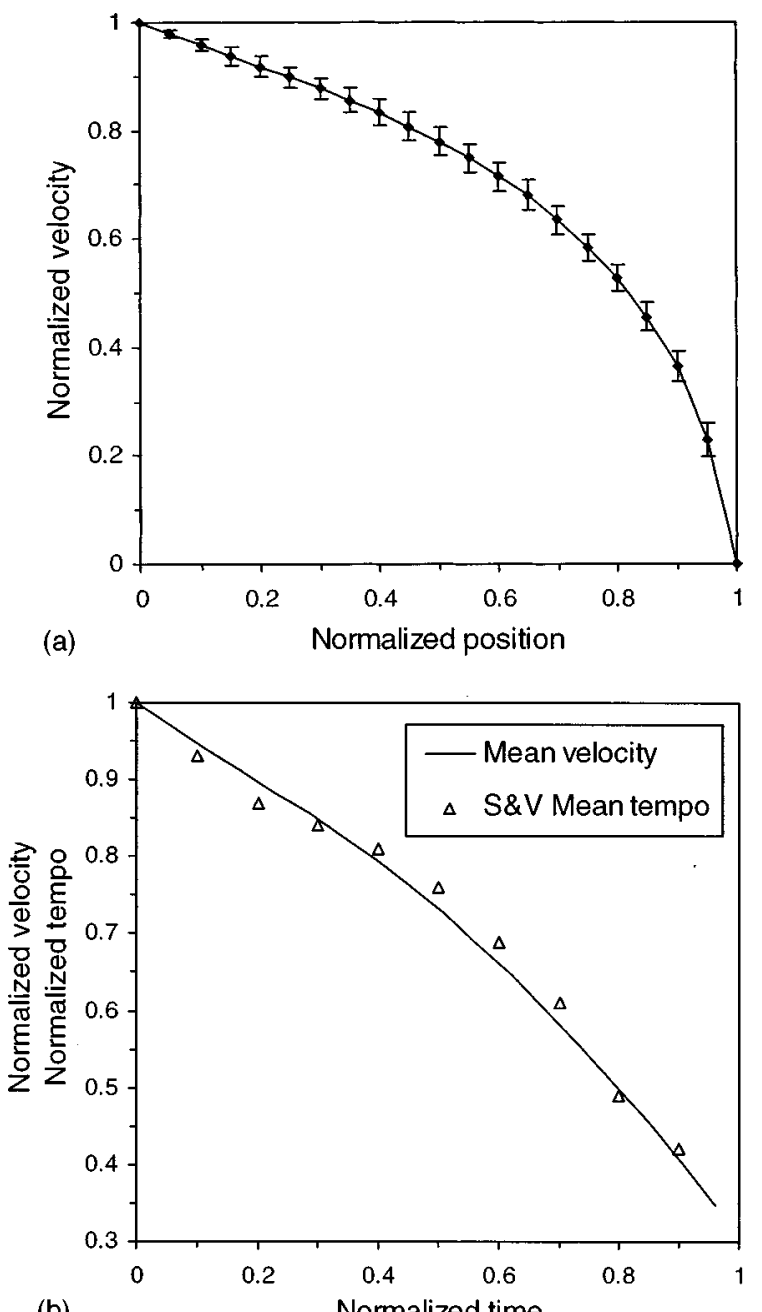

(b)

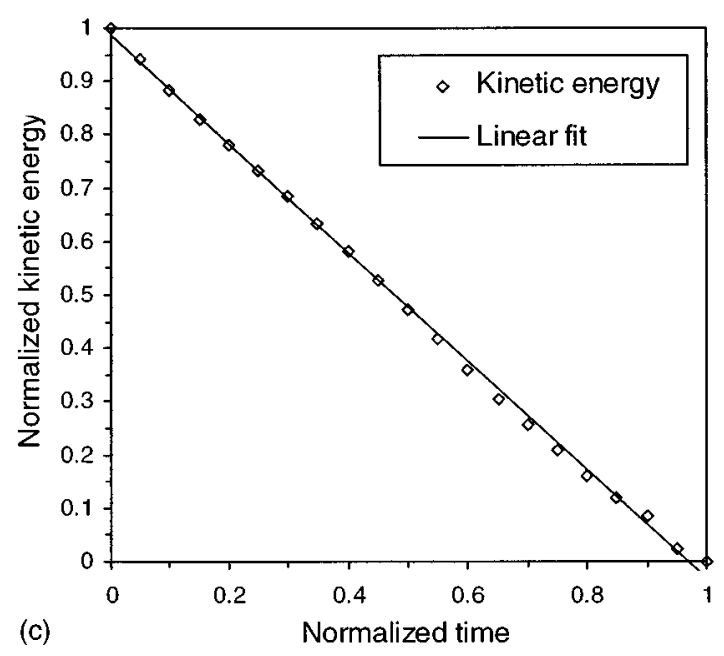

(c)

Normalized time

FIG. 5. (a) Normalized mean velocity for the 12 highest-ranked decelerations as function of normalized score position. The bars mark the 95\%confidence interval. (b) Normalized mean velocity for the 12 highest-ranked decelerations as a function of normalized time (solid line) and mean normalized ritardando tempo versus normalized time according to Sundberg and Verrillo (1980). The former curve was obtained after scaling the time values by a factor of 1.13 , see the text. (c) Squared normalized mean velocity for the 12 highest-ranked decelerations as a function of time. The line shows the best linear fit.

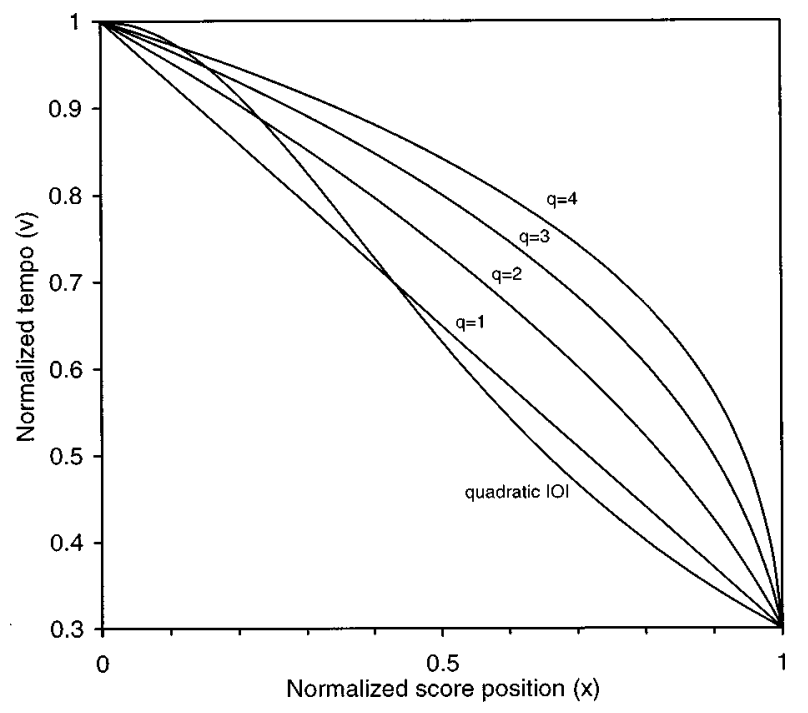

FIG. 6. The ritardando model with four different $q$ values $(q=1,2,3,4)$ and the quadratic IOI function. The final tempo was fixed at $v_{\text {end }}=0.3$. These five curves were used in the listening experiment.

that the kinetic energy is a linear function of time and, since kinetic energy is proportional to velocity squared, velocity will be proportional to a square-root function of time. Let $v$ be velocity (tempo), $x$ be position (score position), then

$$
v=\frac{d x}{d t} \sim \sqrt{t}
$$

Primarily for practical purposes discussed above, $x$ was chosen as the independent variable. Integrating $v$ with respect to $t$, solving for $t$, and substituting $t$ in Eq. (1), we obtain

$$
v(x) \sim x^{1 / 3} .
$$

Thus, velocity (tempo) is proportional to the cubic root of position (score position). Reinspection of the individual $\mathrm{ri}$ tardandi analyzed by Sundberg and Verrillo (1980) revealed variation with regard to the overall curvature. To account for this variation, a curvature parameter $q$ was introduced

$$
v(x) \sim x^{1 / q} .
$$

By changing the constant $q$, a number of different curvatures can be obtained, including the runners' mean deceleration $(q=3)$ as well as the previously mentioned square-root function $(q=2)$.

Unlike velocity in locomotion, the tempo never reaches zero in music, if tempo is defined as the inverse of tone IOI. This implies the need for a second parameter, the final tempo $v_{\text {end }}$. The resulting model of the tempo $(v)$ as a function of score position $(x)$ was defined as

$$
v(x)=\left[1+\left(v_{\mathrm{end}}^{q}-1\right) x\right]^{1 / q} .
$$

Here, tempo and $v_{\text {end }}$ are normalized with respect to the preritardando tempo $v_{\text {pre }}$ and position is normalized with respect to total ritardando length measured in score units. Score position $x=0$ corresponds to the start position of the ritardando and $x=1$ to the onset of the last note. Thus, $v=1$ at $x=0$ and $v=v_{\text {end }}$ at $x=1$. Figure 6 shows the resulting tempo curves for four different $q$ values. The values of $q=1$, $q=2$, and $q=3$ correspond to (a) a linear function of $x$, (b) a square-root function of $x$ (i.e., a linear function of $t$ ), as pro- 
posed earlier by Kronman and Sundberg, and Todd, and (c) the approximation of runners' deceleration, respectively.

The translation from the continuous curve to discrete tones can be realized by integrating the inverse of the tempo function in Eq. (2):

$$
\int \frac{1}{v}=\int \frac{d t}{d x}=\int\left[1+\left(v_{\text {end }}^{q}-1\right) x\right]^{-1 / q} .
$$

Let $k=v_{\text {end }}^{q}-1$. Then, we obtain time as a function of score position

$$
t(x)=\frac{q(1+k x)^{(q-1) / q}-q}{(q-1) k}, \quad q>1 .
$$

The integration constant was determined by setting $t(0)$ $=0$. The IOI of a tone is given by the time difference for the $x$ values corresponding to the onset of the tone $\left(x_{1}\right)$ to the onset of the following tone $\left(x_{2}\right)$.

$$
\begin{aligned}
\mathrm{IOI} & =t\left(x_{2}\right)-t\left(x_{1}\right) \\
& =\frac{q\left(1+k x_{2}\right)^{(q-1) / q}-q\left(1+k x_{1}\right)^{(q-1) / q}}{(q-1) k} .
\end{aligned}
$$

The advantage of using $t(x)$ to determine the tone durations is that it is independent of note values. For example, four sixteenth notes will add up exactly to the duration of one quarter note. It means that Eq. (4) can be applied on each voice in a polyphonic example and at the same time keep the synchrony. This is not the case if IOI is obtained by inverting the tempo value obtained from the continuous tempo curve at, e.g., the onset position of the tone.

The model [Eq. (2)] has the advantage that it is easy to transform, if time instead of position is preferred as the independent variable. Tempo as a function of time can be obtained by solving Eq. (3) for $x$ and substituting the result in Eq. (2)

$$
v(t)=\left[1+\left(v_{\text {end }}^{q-1}-1\right) t\right]^{1 /(q-1)} .
$$

Note that this equation is essentially the same as Eq. (2), with the value of $q$ decreased by one.

\section{A. Perceptual evaluation of the model}

A listening experiment was performed to assess the preferred curvature value $q$ in different music examples. The previously mentioned curve with IOI as a quadratic function of score position was included as an additional alternative.

\section{Stimuli and procedure}

Three different music examples were used, two excerpts from pieces by J. S. Bach and one sequence of two alternating notes a minor second apart, see Table III. In both Bach examples, the ritardando was applied to an unbroken sequence of notes of equal note values. These examples were chosen for evaluating the ritardando in musically realistic contexts, while the minor second example was chosen to attain a minimum of musical content without destroying the perception of the ritardando curve. When informally listening to the examples, the authors found that a simple tone repetition was not enough to differentiate the curvatures.
TABLE III. Music examples used in the listening experiment. The tempo before the ritardando $\left(v_{\text {pre }}\right)$ and at the end $\left(v_{\text {end }}\right)$, normalized with respect to $v_{\text {pre }}$, are also listed.

\begin{tabular}{lccc}
\hline \hline \multicolumn{1}{c}{ Music example } & Abbreviation & $\begin{array}{c}v_{\text {pre }} \\
\text { (shortest notes/s) }\end{array}$ & $v_{\text {end }}$ \\
\hline $\begin{array}{l}\text { J. S. Bach: Eng. Suite 2 Prel., } \\
\text { last 5 meas. }\end{array}$ & E2P & 6.48 & 0.3 \\
$\begin{array}{l}\text { J. S. Bach: Wohltemp. clav. I } \\
\begin{array}{l}\text { Prel. 1, last 5 meas. } \\
\text { minor second sequence }\end{array}\end{array}$ & WIP & 4.47 & 0.4 \\
$\begin{array}{l}\text { (Db4-C4-Db4-C4-Db4 } \\
-\mathrm{C} 4-, \ldots, D b 4), 21 \text { notes total }\end{array}$ & $\mathrm{m} 2$ & 5 & 0.4 \\
\hline \hline
\end{tabular}

Four different $q$ values $(q=1,2,3,4)$ were used for the ritardando model [Eq. (2)]. For the phrasing curve with IOI as a quadratic function of score position, the expression

$$
v(x)=\frac{1}{\left(1 / v_{\text {end }}-1\right) x^{2}+1}, \quad v_{\text {end }}>0
$$

was used, see Fig. 6 .

The pre-ritardando tempo $\left(v_{\text {pre }}\right)$, ritardando length, and final tempo $\left(v_{\text {end }}\right)$ were fixed for each music example throughout the test (see Table III), and determined according to the advice of two professional musicians, Lars Frydén and Monica Thomasson, who listened to different alternatives. In all examples, the ritardando started at the first note onset occurring at least at $1.3 \mathrm{~s}$, as measured in pre-ritardando note duration, preceding the last note.

When informally listening to the examples, it was found that the IOI of the penultimate note was important to the perceived magnitude of the ritardando. The method described above [Eq. (4)] for translation of the continuoustempo function to discrete-note IOI did not allow specification of an exact value for the lengthening of the penultimate note (i.e., if the $v_{\text {end }}=0.4$, the duration of the penultimate will not be $\mathrm{DR}=1 / 0.4)$. Therefore, a second normalization was performed so that the IOI of the penultimate note was set to $1 /\left(v_{\text {end }} * v_{\text {pre }}\right)$.

Special attention also had to be paid to the last note. Its note value is sometimes the same as that of the preceding note, but sometimes considerably longer. In the latter case, no further prolongation of the last note is necessary. On the contrary, such long final notes can even be shortened in a real performance. In the test, the duration of the final note was simply set to 1.25 times the IOI of the penultimate note (m2 example). However, if the last note was already longer than this, it was left unchanged (E2P and WIP examples).

Lacking information about the relation between tempo and dynamics in final ritardandi, the music examples were played on sampler synthesis of a harpsichord (SampleCell). The ritardando model was implemented in the DIRECTOR MUSICES program (Friberg, 1995a) on a Macintosh computer. The examples were recorded on a DAT tape with some artificial reverberation (Yamaha REV7). All judges listened to the tape over earphones adjusted to a comfortable listening level.

The test tape contained 51 examples. The first six examples were selected from the following and were used for 
practice trials only. The remaining 45 examples (5 curves $\times 3$ music examples $\times 3$ repetitions) were presented in random order. The entire test tape took $17 \mathrm{~min}$.

The judges were asked to mark on a 10-cm-long visual analogue scale on an answering sheet how musical they found the performance of the ritardando. The endpoints of the line were labeled "extremely good" and "extremely bad." The listeners were also instructed to try to ignore the performance of the music preceding the ritardando as well as the length of the final note, since these parameters did not change with tempo curvature. The rating values used in the subsequent analysis was defined as the length in $\mathrm{mm}$ from the extremely bad endpoint, i.e., the better ritardando, the higher the rating value.

\section{Judges}

A basic hypothesis was that the ritardando alludes to the stopping of locomotion and hence should be familiar not only to professional musicians playing classical repertoire. Therefore, 19 students were used as judges, all taking the course in music acoustics at the Royal Institute of Technology. After the test, they answered a questionnaire about their musical background. The questionnaire asked if they had experience performing music, if so, what instrument, how many years of performing, how many hours per week, and in which music styles. In addition, they could make any general comment about the test.

According to the answers from the questionnaire, most of the judges could be considered as amateur musicians. Nine judges reported that they had some experience of classical music. One of these had professional experience as a classical musician. Most judges found the test quite difficult, except the professional player. Thus, the task was difficult for listeners lacking previous experience with performance of ritardandi on a professional level.

\section{Results}

Using the judges' ratings as the dependent variable, the results were submitted to a repeated-measures analysis of variance performed by the SUPERANOVA 1.11 program for Macintosh. The within factors were 5 curves $\times 3$ music examples $\times 3$ repetitions. Despite the reported dif-

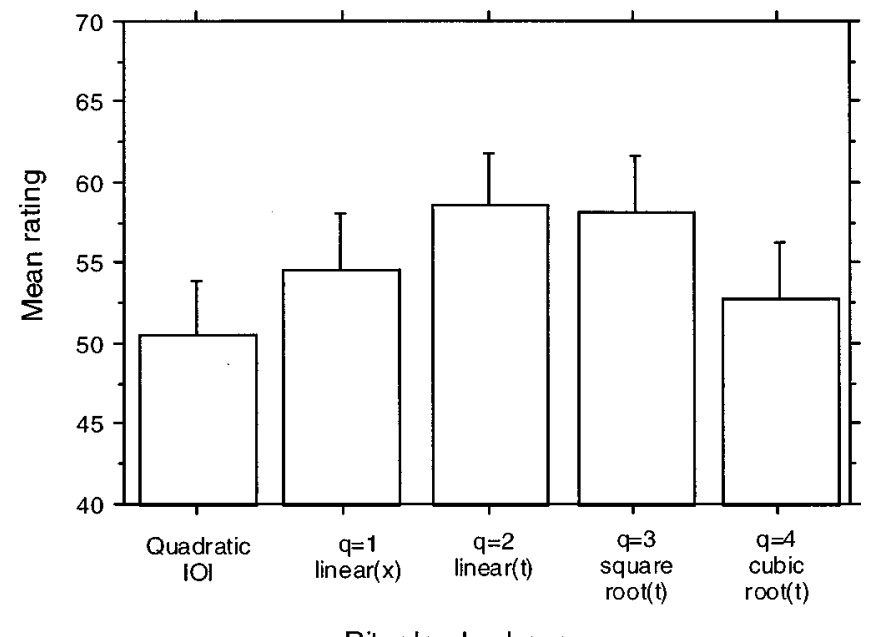

Rit ardando shape

FIG. 7. Overall ratings, averaged across listeners, music examples, and repetitions, of the different curvatures used in the test. The bars indicate the positive parts of a $95 \%$-confidence interval.

ficulty of the test, the main effect of curve was highly significant $(p<0.0001)$, indicating that the judges clearly could differentiate the curves.

The main effect of music example was significant ( $p$ $<0.0001)$; thus, the ritardandi were rated differently depending on the music examples. There was also a significant effect of repetition $(p<0.015)$. No interaction terms were significant. The main effect of music example and repetition disappeared when the $\mathrm{m} 2$ example was excluded from the analysis. The fact that there was a strong effect of repetition only in the case of the $\mathrm{m} 2$ example may indicate that this example had a strange musical content to which, however, the listeners gradually became accustomed.

The mean ratings for the different curvatures are shown in Fig. 7. They reveal that the two curvatures that received the highest ratings originated from the model with $q=2$ and $q=3$. The first value corresponds to the model supported by Kronman and Sundberg (1987) and by Todd (1992, 1995), while the second value corresponds to the curvature derived from runners' decelerations. Both for higher and lower $q$ values and the quadratic IOI, the mean ratings were lower. This was confirmed by a highly significant contrast analysis resulting from a comparison of the cases $q=2$ and $q=3$ with the three remaining alternatives $(p<0.0001)$.

Figure 8, showing the means for each music example

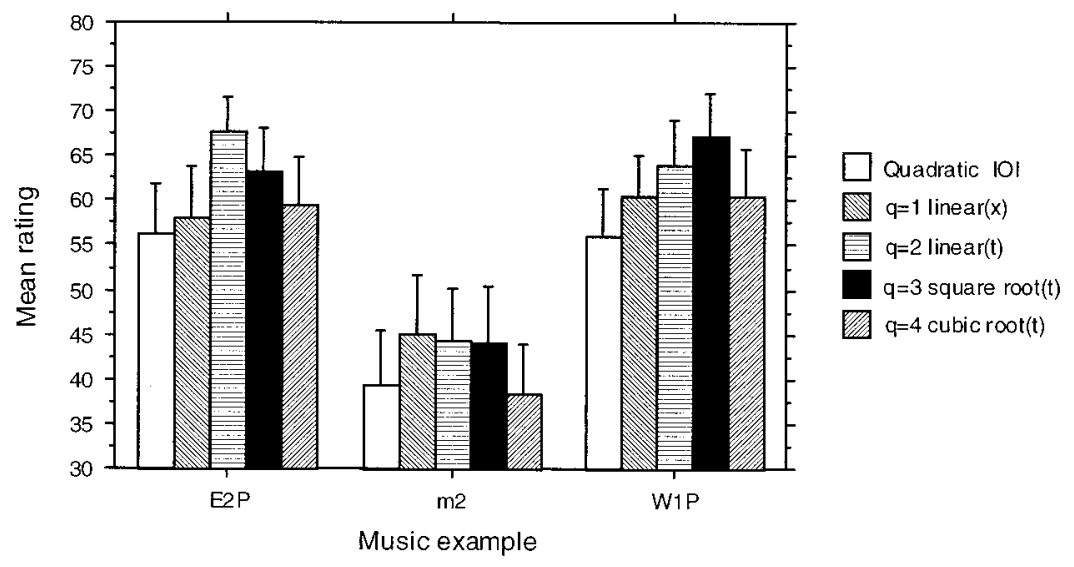

FIG. 8. Mean ratings for the interaction of music example and type of ritardando curve. The bars indicate the positive parts of a $95 \%$-confidence interval. 

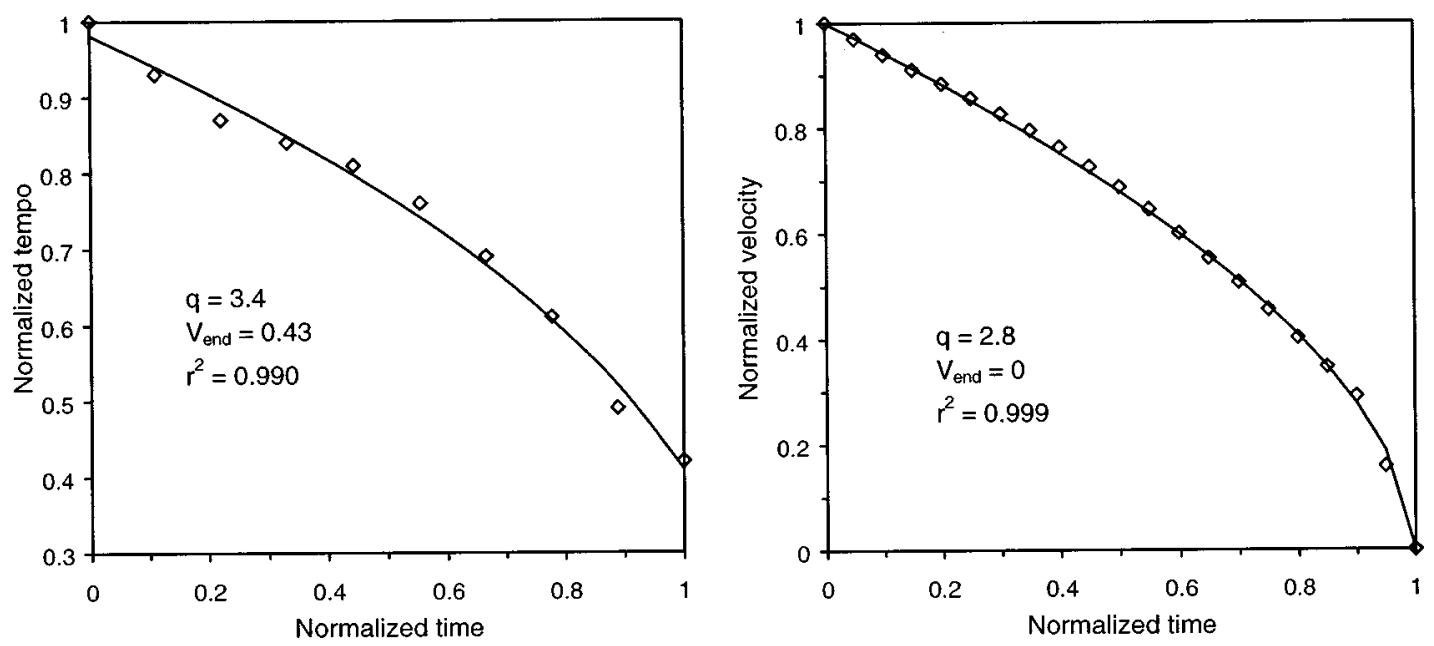

FIG. 9. Left panel: The model (solid line) fitted to the mean ritardando curve (diamonds) from Sundberg and Verrillo. Right panel: The model (solid line) fitted to the mean velocity curve (diamonds) of the runners' deceleration. Note that time rather than position is used as the independent variable. The model-parameter values used and the resulting determination coefficient are also shown.

and curve, offers a more detailed representation of the results. First, it can be noted that for all curves the minor second example was rated much lower than the other examples. Obviously, despite the instruction to concentrate on the ritardando, the judges were unable to disregard the musical context. Second, the maximum rating was obtained for the $q=2$ curve in the E2P example and for the $q=3$ curve in the WIP example. This suggests that the optimum curve is dependent on the music example. A contrast analysis comparing $q=2$ for E2P and $q=3$ for WIP with $q=3$ for E2P and $q=2$ for WIP showed a barely significant difference $(p<0.04)$. Further testing with more-skilled listeners is needed to further confirm this hypothesis.

\section{B. Matching the model to measurements}

In a previous section, the runners' deceleration was found to fit well with the average ritardando curve. Here, the ability of the model to fit performed mean and individual ritardandi as well as runner's mean and individual decelerations will be examined.

The $q$ and $v_{\text {end }}$ parameters in the model, defined in Eq. (2), were varied by means of the solver function in Microsoft EXCEL 7.0, such that the sum of the squared distances between the model and the measured data was minimized. The normalization of measured ritardandi and decelerations were done relative to the first value. It means that a slight offset of the first value affects all remaining values. Therefore, an additional third parameter, $v_{\text {offset }}$, was varied in the optimizing process, which added a constant to the model.

\section{Average curves}

As an initial check of previous results, the model was fitted to the curves for the mean ritardando and the mean deceleration, see Fig. 9. The fit is good in both cases. In the case of the deceleration curve, however, this was expected, as the model was originally derived from this curve. Figure 9 also specifies the optimal values of the parameters $q$ and $v_{\text {end }}$. For both cases, the $q$ values were close to 3 .

\section{Individual ritardandi measured by Sundberg and Verrillo}

The model was also fitted to individual ritardandi taken from the original raw data by Sundberg and Verrillo. In a ritardando, local lengthenings and shortenings of individual tones frequently occur, depending on musical context. Such local departures from the main ritardando curve increase the scatter in the fitted-parameter values. Therefore, to improve the reliability of the fitted-curvature data, only ritardandi with a smooth shape were selected, which contained a minimum of individual note departures. Thus, only the performances with at least six consecutive final notes with monotonically increasing IOIs were used. In this way, 12 final ritardandi were selected out of a possible total of 22 , all by J. S. Bach, see Table IV. Apart from the present model, two other alternatives were tried. As note IOI is frequently assumed to be a quadratic function of score position, one alternative was a quadratic polynomial in which the three parameters were fitted to measured IOIs (henceforth, quadratic IOI). In the other alternative, a similar quadratic function was used to approximate the tempo rather than note IOI (henceforth, quadratic tempo). The fitted-model parameter values and the resulting determination coefficients $\left(r^{2}\right)$ for the 12 examples are listed in Table IV. The model fitted to the 12 examples is plotted in the Appendix (Fig. A1). A comparison of the model and the two alternatives fitted to the first music example (WIP) is shown in Fig. 10. Notice that the quadratic IOI has the undesired property of an initial tempo increase. A more realistic model for the quadratic IOI would be to restrict the tempo to be decreasing. This would make for an even poorer fit.

As shown in Table IV, the mean $q$ value $(q=2.8)$ is very close to the initial value of $q=3$ that was derived from the runners' decelerations. Note that example WIP was also used in the listening experiment above. It received a $q$ value of 2.5 that is just in the middle of the listeners' preference, as seen in Fig. 7. However, $q$ varied substantially between examples. Indeed, two examples yielded $q=1.2$, i.e., the tempo decreased almost linearly with score position, and in three examples, $q$ exceeded the value of four. Such low and high 
TABLE IV. Model parameters $\left(q, v_{\text {end }}, v_{\text {offset }}\right)$ producing the best fit of individual ritardandi for 12 music examples measured by Sundberg and Verrillo (1980). The number of notes included in the ritardando is listed in the second column. The resulting determination coefficients are given in the column marked Model. The two rightmost columns show the determination coefficients $r^{2}$ obtained for the two alternatives tried, the quadratic interonset interval (IOI), and the quadratic tempo (see the text).

\begin{tabular}{|c|c|c|c|c|c|c|c|}
\hline \multirow[b]{2}{*}{ Music examples } & \multirow[b]{2}{*}{ Notes } & \multicolumn{3}{|c|}{ Fitted-model parameters } & \multicolumn{3}{|c|}{ Determination coefficients $\left(r^{2}\right)$} \\
\hline & & $q$ & $v_{\mathrm{end}}$ & $v_{\text {offset }}$ & Model & Quadratic IOI & Quadratic tempo \\
\hline W. clav I Prel. 1 (WIP) & 10 & 2.5 & 0.32 & 0.005 & 0.982 & 0.927 & 0.975 \\
\hline W. clav II Prel. 1 (WP1) & 8 & 2.1 & 0.37 & 0.0001 & 0.980 & 0.989 & 0.983 \\
\hline W. clav II Prel. 2 (WP2) & 7 & 2.4 & 0.51 & 0.03 & 0.967 & 0.989 & 0.977 \\
\hline W. clav II Fug. 3 (WF3) & 6 & 1.2 & 0.48 & -0.03 & 0.969 & 0.951 & 0.968 \\
\hline W. clav II Fug. 5 (WF5a) & 7 & 4.2 & 0.50 & 0.01 & 0.995 & 0.970 & 0.995 \\
\hline W. clav II Fug. 5 (WF5b) & 8 & 2.6 & 0.37 & -0.02 & 0.975 & 0.919 & 0.966 \\
\hline Eng. Suite 1 Allem. (E1A) & 6 & 2.0 & 0.45 & -0.03 & 0.982 & 0.943 & 0.978 \\
\hline Eng. Suite 2 Allem. (E2A) & 11 & 3.7 & 0.37 & 0.02 & 0.975 & 0.927 & 0.980 \\
\hline Fr. Suite 4 Cour. (F4C) & 6 & 4.2 & 0.50 & 0.02 & 0.991 & 0.959 & 0.986 \\
\hline Fr. Suite 6 Allem. (F6A) & 7 & 2.4 & 0.45 & 0.02 & 0.981 & 0.965 & 0.979 \\
\hline Fr. Suite 6 Cour. (F6C) & 7 & 5.0 & 0.46 & -0.008 & 0.994 & 0.902 & 0.959 \\
\hline Italian Conc. Mvt. 3 (IC3) & 7 & 1.2 & 0.34 & -0.01 & 0.970 & 0.961 & 0.970 \\
\hline Mean & & 2.8 & 0.43 & 0.001 & 0.980 & 0.950 & 0.976 \\
\hline s.d. & & 1.2 & 0.07 & 0.02 & 0.01 & 0.03 & 0.01 \\
\hline
\end{tabular}

values of $q$ received low ratings in the listening test above (using other examples), yet were obviously acceptable in these performances. This supports the assumption above that the optimal ritardando curve depends on some characteristics of the music example, e.g., musical structure or tempo.

The final tempo $v_{\text {end }}$ varied comparatively less, between $32 \%$ and $51 \%$ of the initial tempo. Desain and Honing (1996) suggested that the final tempo might be dependent on the global tempo. However, no indication of this was found in the present data. There was no correlation between the final tempo $\left(v_{\text {end }}\right)$ and the pre-ritardando tempo $(\bar{T})$ as defined by Sundberg and Verrillo $(r=-0.02)$.

Our model produced the highest mean correlation with the measurements (mean $r^{2}=0.98$ ). A $t$-test comparing the mean $r^{2}$ for the model and the mean $r^{2}$ for the quadratic IOI alternative yielded a significant difference $(p<0.003)$ : this means that, on average, the model approximated the measured ritardandi better than the quadratic IOI.

The correlation was slightly better for the model than for the quadratic tempo, but this difference was not significant. With regard to the shortest ritardandi, consisting of no more than six notes, it could be argued that many slightly curved functions would offer a good fit. Longer ritardandi, on the other hand, often exhibited a more pronounced tempo decrease at the end than in the beginning. The model also accounted for such cases provided a relatively high value of $q$ was used, see Fig. 11 (this example is not included in Table IV). The quadratic-tempo function, however, failed to produce the characteristic increased curvature toward the end of the ritardando.

\section{Individual ritardandi measured by Feldman, Epstein, and Richards}

Feldman et al. (1992) measured two ritardandi in performances of orchestral music. These ritardandi occurred within the pieces, i.e., not in a final position. Also, they were clearly longer than the final ritardandi considered above, and were measured at the beat level instead at the note level. The three functions presented above were fitted to their example, which had the smoother shape, see Fig. 12 (sample 1: A. Dvořák, Slavonic dance, op 48:8, measures 243-272). Despite the difference in style, length, and context, the result is similar to that observed for the ritardando shown in Fig. 11.
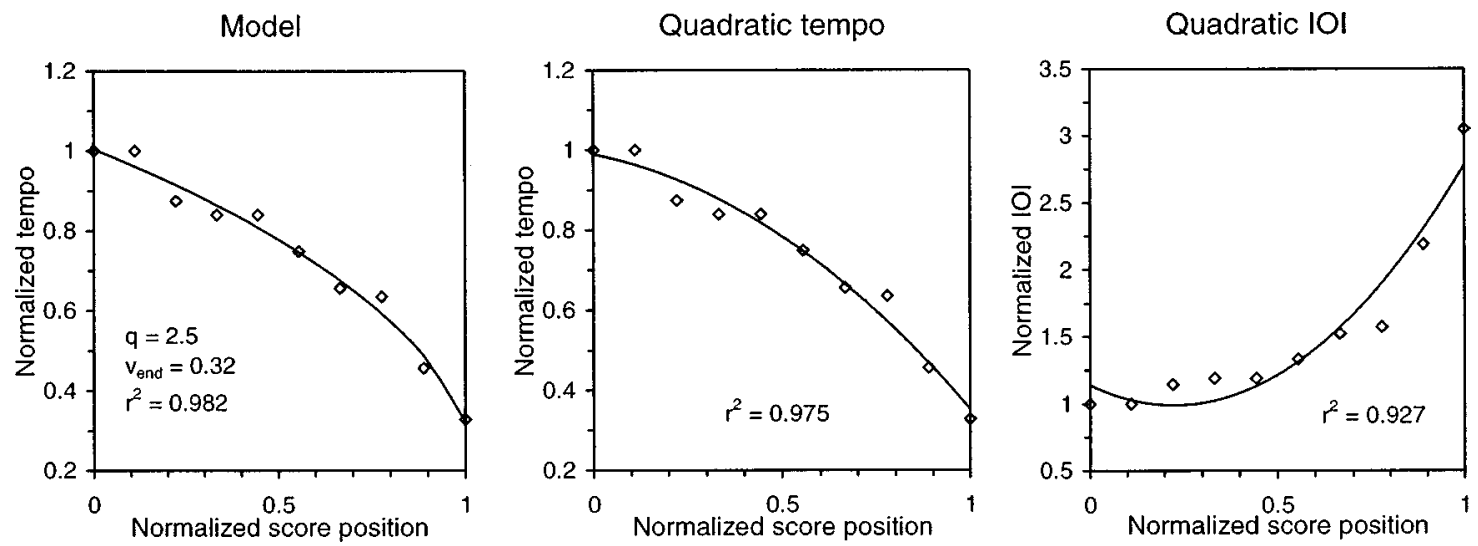

FIG. 10. Model (left), quadratic tempo (middle), and quadratic IOI (right) functions fitted to the ritardando data from music example WIP. The model parameters used are presented in Table IV. 
Model

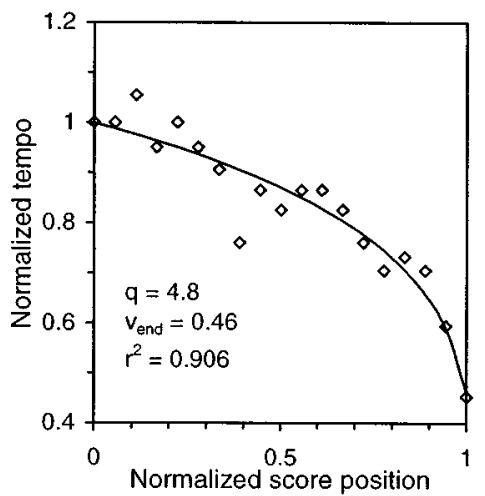

Quadratic tempo

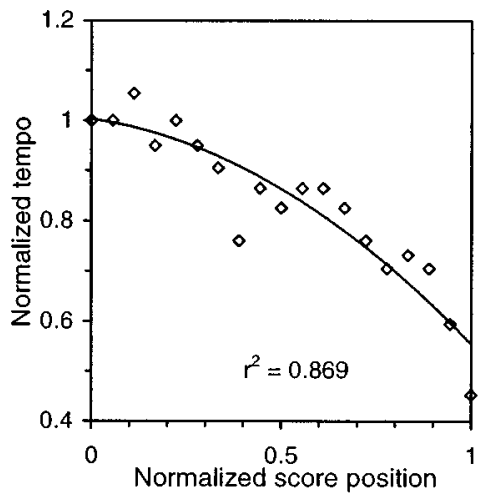

Quadratic IOI

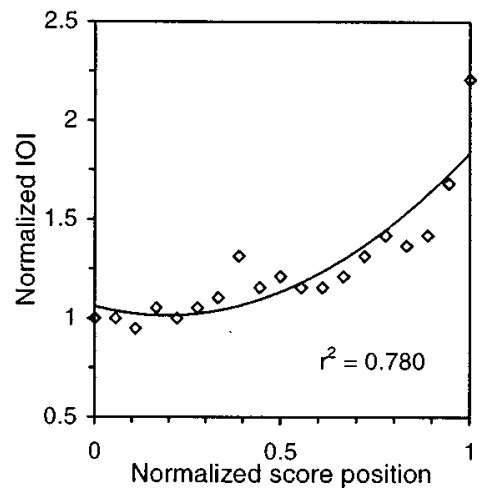

FIG. 11. Model (left), quadratic tempo (middle), and quadratic IOI (right) functions fitted to a comparatively long ritardando measured in a performance of music example WP 19. This example was not included in Table IV. Note that the increased curvature in the end of the ritardando could not be modeled with a quadratic-tempo function.

Again, the model produced the best fit, slightly better than that of the quadratic-tempo function, especially in the end of the ritardando. The fit obtained with the quadratic IOI was the worst. It is noteworthy that Feldman et al. would have obtained much better fits if they had applied quadratic or cubic functions to tempo as a function of time (as they had suggested in the first part of their paper) rather than to IOI of score position.

\section{Individual decelerations of runners}

Table $\mathrm{V}$ presents the model and a quadratic-velocity function fitted to each of the 16 decelerations in Table II. The model fitted to the 16 examples is plotted in the Appendix (Fig. A2). According to the determination coefficients, also shown in Table $\mathrm{V}$, the model matched the data better for the 12 highest-ranked decelerations. A two-tailed $t$-test revealed that the means of the determination coefficients $r^{2}$ differed significantly $(p<0.02)$ between the model and the quadratic-velocity function.

For the four decelerations that were discarded for reasons of low visual rating (Table V, rank 13-16), the determination coefficients for the model were significantly lower (two-tailed $t$-test, $p<0.005$ ) than those of the group of 12 , higher-rated decelerations (mean $r^{2}=0.975$ and 0.990 , respectively). On the other hand, when fitting the quadratic function to these four decelerations, no significant change of $r^{2}$ was observed (mean $r^{2}=0.979$ and 0.978 , respectively).
Thus, a high rating in the visual experiment corresponded to a good fit of the model, but this correspondence was not found if quadratic velocity was used.

\section{GENERAL DISCUSSION}

In discussing the model proposed here for descriptions of final ritardandi and runners' decelerations, certain limitations should be kept in mind. The start of the final ritardando is difficult to identify in measurements, particularly in cases of a smooth onset. Perceptually a smooth onset of the ritardando is important, as mentioned. For example, informal listening tests revealed that the onset of the ritardando was far too abrupt when a linear tempo function of score position was applied. For the examples in Table IV, we identified the ritardando onset by means of a rigorously applied rule; that all tones after the onset must show a progressively decreasing tempo. This rule may yield delays of the point of onset in some cases, which leads to an underestimation of the value of $q$.

There are also other limitations. The perception of a final ritardando may depend not only on tempo changes, but also on dynamic changes. This parameter was not analyzed in the present investigation. Another important factor is its total length. What is considered an appropriate ritardando may also depend on the instrument played. In view of the analogies suggested by our findings between music and lo-
Model

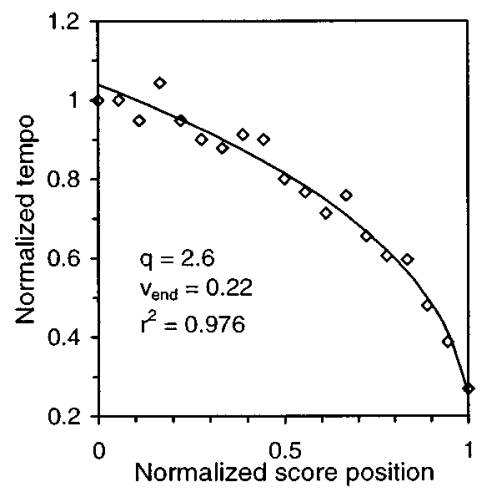

Quadratic tempo

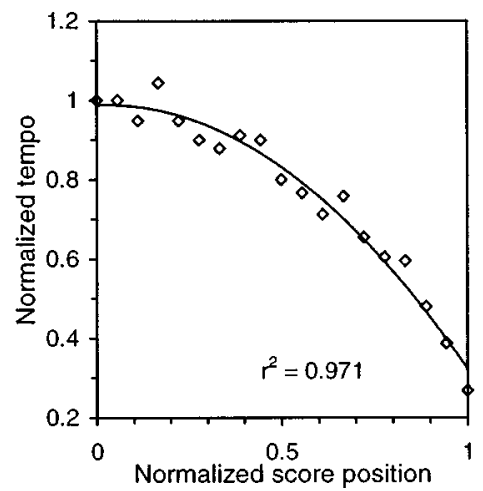

Quadratic IOI

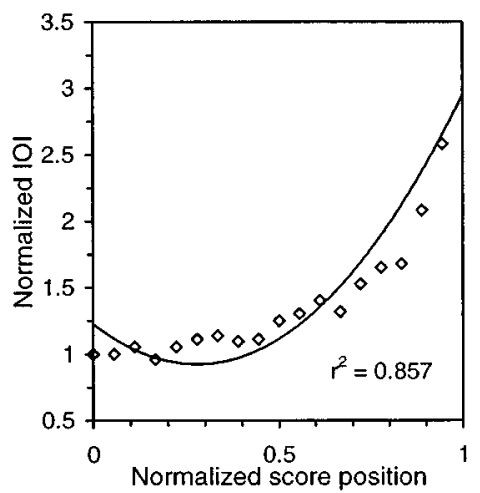

FIG. 12. Model (left), quadratic tempo (middle), and quadratic IOI (right) functions fitted to a ritardando measured by Feldman et al. (1992). 
TABLE V. Model parameters $\left(q, v_{\text {end }}, v_{\text {offset }}\right)$ producing the best fit of 16 individual decelerations produced by the dancers. The decelerations are listed in rank order according to the mean rating of aesthetic quality. The resulting determination coefficients are given in the column marked Model. The two rightmost columns show the determination coefficients $r^{2}$ obtained for the model and for the quadratic velocity alternative. The first 12 decelerations (Rank 1-12) were used for calculating the average deceleration pattern.

\begin{tabular}{|c|c|c|c|c|c|}
\hline \multirow{2}{*}{$\frac{\text { Deceleration }}{\text { Rank }}$} & \multicolumn{3}{|c|}{ Fitted model parameters } & \multicolumn{2}{|c|}{ Determination coefficient $\left(r^{2}\right)$} \\
\hline & $q$ & $v_{\text {end }}$ & $v_{\text {offset }}$ & Model & Quadratic velocity \\
\hline 1 & 2.7 & 0.25 & -0.02 & 0.989 & 0.975 \\
\hline 2 & 2.7 & 0.33 & -0.02 & 0.991 & 0.976 \\
\hline 3 & 3.4 & 0.20 & 0.01 & 0.978 & 0.939 \\
\hline 4 & 3.3 & 0.39 & 0.03 & 0.980 & 0.991 \\
\hline 5 & 2.9 & 0.27 & 0.005 & 0.994 & 0.985 \\
\hline 6 & 1.8 & 0.36 & 0.01 & 0.999 & 0.999 \\
\hline 7 & 3.2 & 0.33 & 0.003 & 0.985 & 0.978 \\
\hline 8 & 2.1 & 0.20 & 0.03 & 0.990 & 0.998 \\
\hline 9 & 2.7 & 0.13 & -0.006 & 0.996 & 0.962 \\
\hline 10 & 2.7 & 0.31 & 0.003 & 0.988 & 0.976 \\
\hline 11 & 3.4 & 0.32 & 0.01 & 0.995 & 0.981 \\
\hline 12 & 2.7 & 0.21 & -0.02 & 0.996 & 0.973 \\
\hline Mean $(1-12)$ & 2.8 & 0.27 & 0.004 & 0.990 & 0.978 \\
\hline s.d. & 0.5 & 0.08 & 0.02 & 0.006 & 0.016 \\
\hline 13 & 2.5 & 0.08 & 0.07 & 0.965 & 0.966 \\
\hline 14 & 3.0 & 0.35 & 0.007 & 0.992 & 0.989 \\
\hline 15 & 2.7 & 0.16 & 0.07 & 0.974 & 0.989 \\
\hline 16 & 2.0 & 0.29 & 0.03 & 0.968 & 0.972 \\
\hline Mean (13-16) & 2.6 & 0.22 & 0.05 & 0.975 & 0.979 \\
\hline s.d. & 0.4 & 0.12 & 0.03 & 0.012 & 0.012 \\
\hline
\end{tabular}

comotion, it would be tempting to explore possible musical equivalents of, e.g., inertia and its effect on the final ritardando.

It appeared that it was more difficult for the listeners to differentiate the tempo curves in a musically unnatural example. In selecting the music examples for the listening test, the authors frequently noted that the perceived character of the different curves was well exposed by real music examples but almost impossible to distinguish in unrealistic, simple examples, such as a sequence of tone repetitions. On the other hand, the tempo curve seems to originate from the paramount experience of locomotion. Maybe the musical unnaturalness of such examples distracts the listener's attention from the tempo curve. In any event, realistic musical examples seem crucial for a correct evaluation of any music performance model.

The quadratic IOI curve gave the poorest results, both in the listening experiment and in the curve fittings. This was surprising, since it had been successfully applied to describe tempo curves associated with phrasing (Todd, 1985; Repp, 1992b; Friberg, 1995b; Penel and Drake, 1998). Transformed to tempo as a function of score position, the quadratic IOI curve assumes a shape characterized by a gradual decrease of the curve steepness, as was illustrated in Fig. 1. If translated to velocity in locomotion, such a curve shape would imply that the runner refrains from spending energy on reduction of speed toward the end of the deceleration process, thus suggesting a continuation of the movement. This message appears quite appropriate at phrase endings; the music will continue beyond the phrase boundary. Indeed, according to informal listening tests the impression of an approaching final stop disappeared when the quadratic IOI curve was used for final ritardandi; it was no more obvious that the last tone really was the last.

A factor of possible relevance to our results is musical style. The measurements and fittings in which the quadratic IOI was used mainly concerned romantic classical music, while the present final ritardando model was mostly tested on baroque music [except for sample 1 from Feldman et al. (1992), which was composed by Dvorák]. It is also possible that the poor success of the quadratic IOI alternative reflected that these two music styles are associated with different types of motion.

Surprisingly, we found two cases of $q=1.2$ in the fittings of the individual ritardandi, i.e., the tempo decreased almost linearly with score position. Such a curve $(q=1)$ was rated low in the listening experiment (applied on different examples). Also, when applied in informal tests to a variety of music examples, this curve sounded musically unacceptable. As with the quadratic IOI one problem was that the piece did not appear to approach a final stop during the last part of the ritardando.

Why does our model work? The two cases $q=2$ and $q=3$, which received the highest ratings in the listening experiment, have a very simple form in terms of locomotion; the former implies that the braking force is constant while the latter implies that the braking power is constant. Such simple relations would facilitate prediction of the point of final stop.

The deceleration power was found to be approximately constant throughout the entire deceleration process. Thus, the dancers' velocity patterns appeared to be well planned, which should enable a spectator to predict the point of final stop. The similarity between the velocity curves and the tempo curves for the ritardando supports the hypothesis that the ritardando is an allusion to the stopping of running. Predictability of the instant at which the final tone appears in a piece of music is important not only to the players but probably also to the listeners. It is possible that the time of appearance of the final tone becomes predictable in music listening because of the similarity between the final ritardando and a runner's deceleration curve.

This investigation explored one possibility to analyze the relations between music and motion, but there are also other alternatives. For example, comparisons between handmovement patterns, such as during conducting, and local tempo patterns might offer further interesting insights into the similarities between motion and music.

\section{CONCLUSION}

The mean instant velocity during runners' decelerations, perceived as aesthetically pleasing, can be accurately described by a square-root function of time. This implies that the runner is supplying a constant amount of deceleration power throughout the entire deceleration process. Such a deceleration pattern is strikingly similar to a curve representing the mean instant tempo versus time in final ritardandi of musical performances.

A model of the final ritardando was derived from runners' mean-deceleration curve, assuming constant decelera- 
tion power. By introducing two main parameters, $q$ for curvature and $v_{\text {end }}$ for the end value, this model could well describe the average tempo curve in final ritardandi, the average velocity curve of runners' decelerations, as well as individual final ritardandi, and runners' individual decelerations. These findings substantiate the common assumption that locomotion and music are related.

A perceptual evaluation of the model indicated that listeners preferred $q$ values equal to 2 and 3 . The case of $q$ $=3$ corresponds to the average velocity of the runners' decelerations, while $q=2$ corresponds to a previously proposed model for final ritardandi (Kronman and Sundberg, 1987) as well as to Todd's model for phrase-related tempo curves (Todd, 1992, 1995). However, in some individual ritardandi the curvature parameter $q$ deviated from the values preferred in the listening experiment, thus suggesting influence of musical factors. Previous investigations have revealed that the tempo variation related to musical phrases can be described by expressing the IOI duration as a quadratic function of score position. This alternative yielded poor results, both in the listening experiment and in the attempts to match individual measured ritardandi.

\section{ACKNOWLEDGMENTS}

We are indebted to Alf Thorstensson and Johnny Olsson, who provided the hall for the recordings and assisted in the measurements of the runners. Ulla Hellqvist, the Royal College of Music, Stockholm, is gratefully acknowledged for performing the initial evaluation of the decelerations. Sofia Dahl, Jenny Iwarsson, and Bo Lantz assisted in the analysis of the video data. We also gratefully acknowledge the kind assistance from Hans Norén and his colleagues at the Swedish Radio, Stockholm, for providing a harpsichord in excellent condition for the recording of the tones that were used in the listening experiment. Lars Frydén and Monica Thomasson kindly assisted in the selection of music examples and model parameters for the listening experiment. This work was supported by the Swedish National Council for Research in the Humanities and Social Sciences, and the Bank of Sweden Tercentenary Foundation.

\section{APPENDIX}
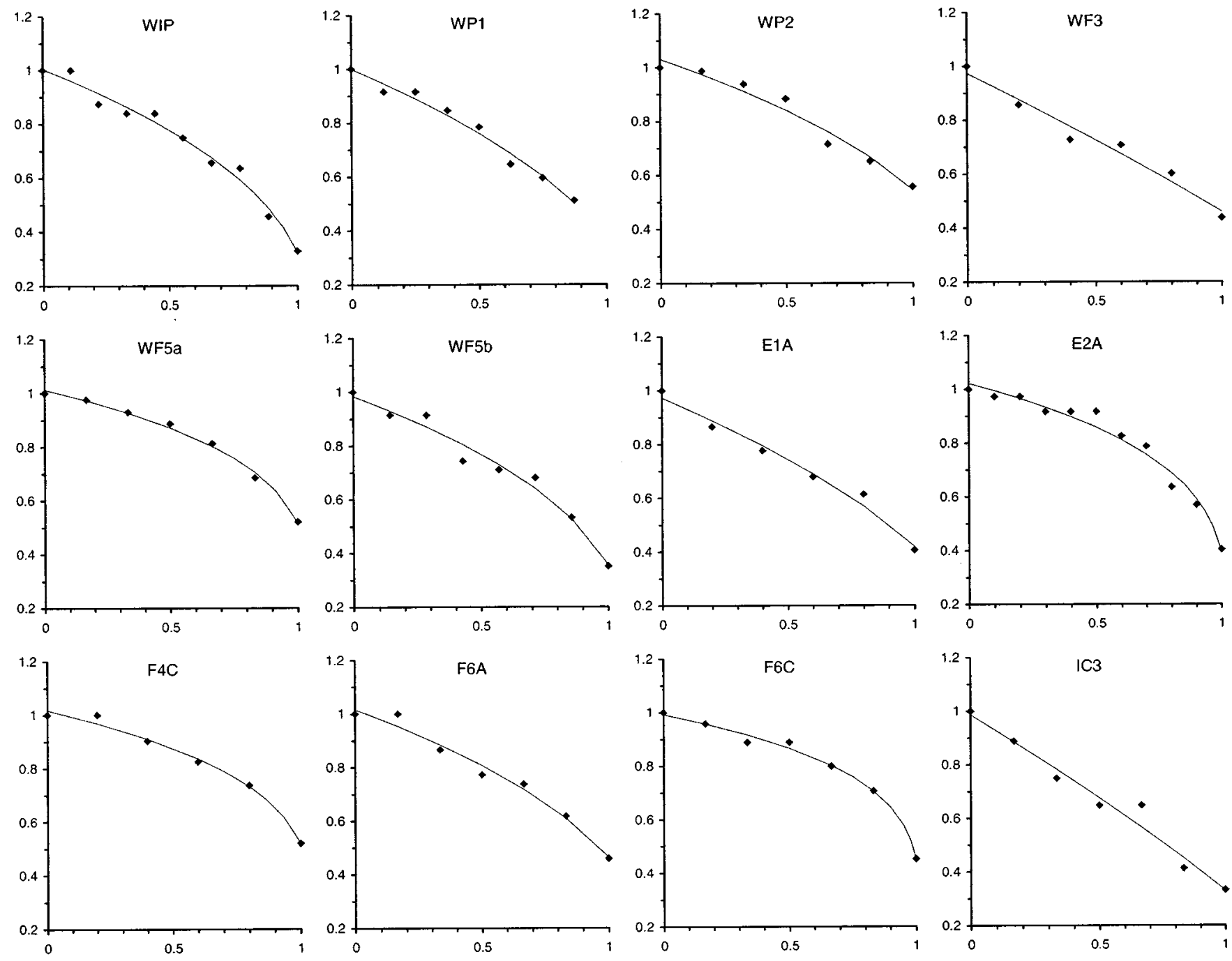

FIG. A1. The model (solid line) fitted to each of the 12 final ritardandi (diamonds) selected from the Sundberg and Verrillo investigation. The vertical axis represents normalized tempo and the horizontal axis, normalized score position. The model parameters used are presented in Table IV. 

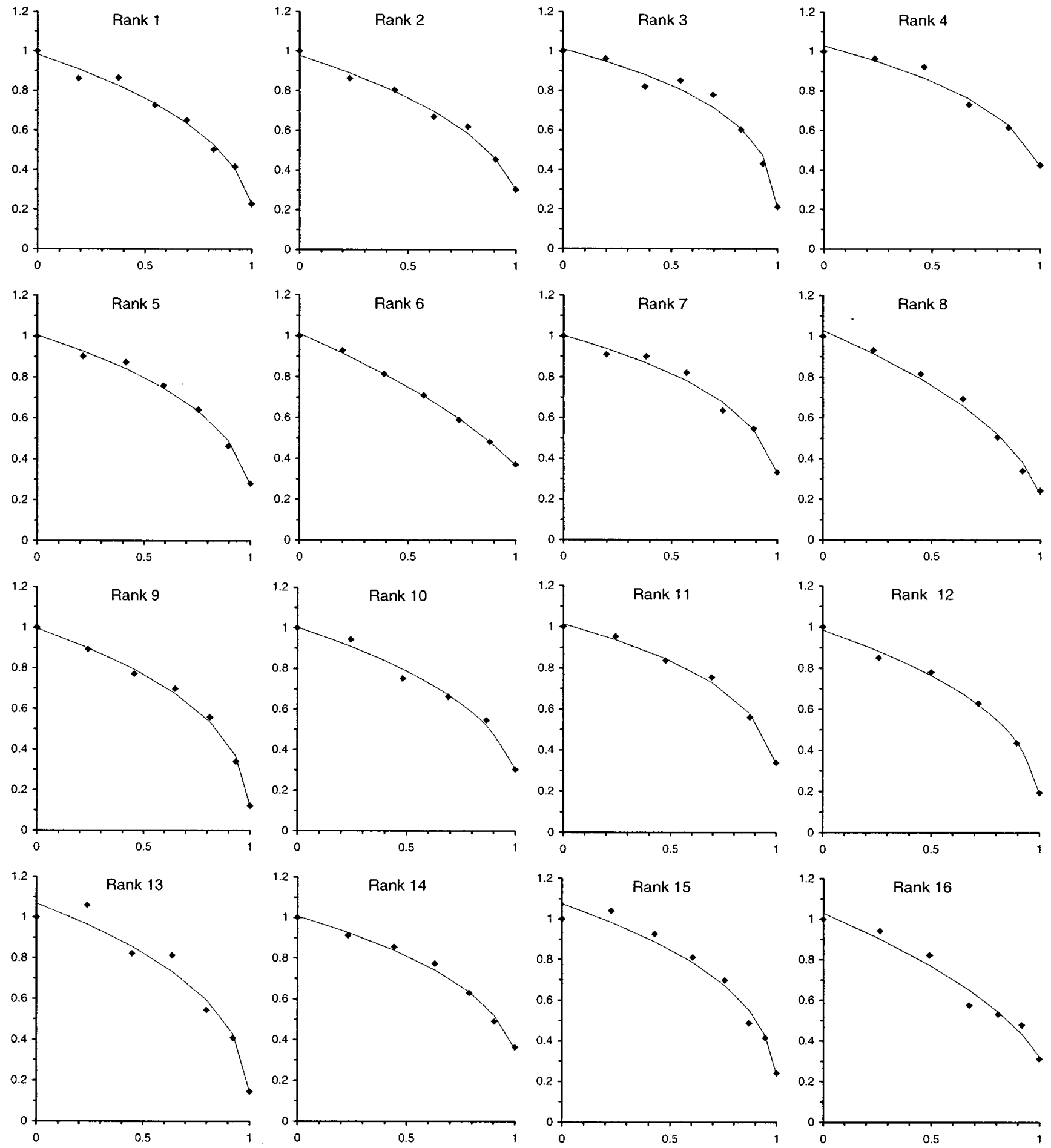

FIG. A2. The model (solid line) fitted to each of the 16 decelerations (diamonds) used in the visual rating experiment. The vertical axis represents normalized velocity and the horizontal axis, normalized position. The model parameters are presented in Table V.

Ae, M., Ito, A., and Suzuki, M. (1992). “The men's 100 metres,' New Studies Athlet. 7(1), 47-52.

Alexander, R. McN. (1995). "Simple models of human movement," Appl. Mech. Rev. 48, 461-469.

Alexander, R. McN., and Maloiy, G. M. O. (1984). "'Stride length and stride frequencies of primates," J. Zool. 202, 577-582.

Cavagna, G. A., Franzetti, P., Heglund, N. C., and Willems, P. (1988). “The determinants of the step frequency in running, trotting and hopping in man and other vertebrates," J. Physiol. 399, 81-92.

Desain, P., and Honing, H. (1996). "Physical motion as a metaphor for timing in music: the final ritard," in Proceedings of the 1996 International Computer Music Conference (ICMA, San Francisco, CA), pp. 458-460.

Feldman, J., Epstein, D., and Whitman, R. (1992). "Force dynamics of tempo change in music," Music Percept. 10(2), 185-203.

Friberg, A. (1995a). "A Quantitative Rule System for Musical Performance," Doctoral dissertation, Royal Institute of Technology, Sweden.

Friberg, A. (1995b). "Matching the rule parameters of phrase arch to performances of 'Träumerei': A preliminary study," in Proceedings of the KTH Symposium on Grammars for Music Performance, May 27, 1995, edited by A. Friberg and J. Sundberg (Speech, Music and Hearing, Royal Institute of Technology, Stockholm), pp. 37-44.

Kronman, U., and Sundberg, J. (1987). "Is the musical ritardando an allusion to physical motion?"' in Action and Perception in Rhythm and Music, edited by A. Gabrielsson (Royal Swedish Academy of Music, Stockholm), Publication No. 55, pp. 57-68.

Longuet-Higgins, H. C., and Lisle, E. R. (1989). "Modelling music cogni- 
tion,', Contemp. Music Rev. 3, 15-27.

Margaria, R. (1976). Biomechanics and Energetics of Muscular Exercise (Clarendon, Oxford).

Nilsson, J., and Thorstensson, A. (1987). "Adaptility in frequency and amplitude of leg movements during human locomotion at different speeds,", Acta Physiol. Scand. 129, 107-114.

Penel, A., and Drake, C. (1998). "Sources of timing variations in music performance: A psychological segmentation model,' Psychol. Res. 61, 12-32.

Repp, B. H. (1992a). "'Diversity and commonality in music performance: An analysis of timing microstructure in Schumann's 'Träumerei,',' J. Acoust. Soc. Am. 92, 2546-2568.

Repp, B. H. (1992b). "A constraint on the expressive timing of a melodic gesture: Evidence from performance and aesthetic judgement," Music Percept. 10(2), 221-242.

Repp, B. H. (1993). "Music as motion: a synopsis of Alexander Truslit's (1938) 'Gestaltung und Bewegung in der Musik,',' Psychol. Music 21(1), $48-72$.

Sederholm, E., McAllister, A., Sundberg, J., and Dahlkvist, J. (1993). "Perceptual analysis of child hoarseness using continuous scales,' Scand. J. Log. Phon. 18, 73-82.

Shove, P., and Repp, B. H. (1995). "Musical motion and performance: theoretical and empirical perspectives," in The Practice of Performance: Studies in Musical Interpretation, edited by J. Rink (Cambridge U.P., Cambridge), 55-83.

Sundberg, J. (1988). "Computer synthesis of music performance," in Generative Processes in Music, edited by J. A. Sloboda (Clarendon, Oxford), 52-69.

Sundberg, J., Friberg, A., and Frydén, L. (1992). "Music and locomotion. A study of the perception of tones with level envelopes replicating force patterns of walking,' KTH Speech Transmission Laboratory Quarterly Progress and Status Report, 4/1992, 109-122.

Sundberg, J., and Verrillo, V. (1980). "On the anatomy of the ritard: A study of timing in music,', J. Acoust. Soc. Am. 68, 772-779.

Todd, J. T. (1983). "Perception of gait,', J. Exp. Psychol.: Human Percept. Performance 9(1), 31-42.

Todd, N. P. McA. (1985). "A model of expressive timing in tonal music,", Music Percept. 3, 33-58.

Todd, N. P. McA. (1992). "The dynamics of dynamics: A model of musical expression,', J. Acoust. Soc. Am. 91, 3540-3550.

Todd, N. P. McA. (1995). "The kinematics of musical expression," J. Acoust. Soc. Am. 97, 1940-1949.

Truslit, A. (1938). Gestaltung und Bewegung in der Musik (Chr. Friedrich Vieweg, Berlin-Licherfelde). 